\title{
Vascular Endothelial Growth Factor Signaling Regulates the Segregation of Artery and Vein via ERK Activity during Vascular Development
}

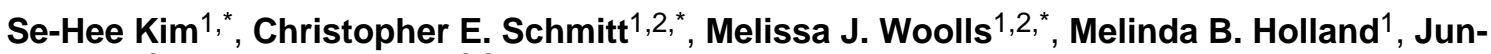 \\ Dae Kim², and Suk-Won Jin ${ }^{2,3}$ \\ ${ }^{1}$ McAllister Heart Institute, and Curriculum in Genetics and Molecular Biology, University of North \\ Carolina at Chapel Hill, Chapel Hill, NC, 27599, USA \\ ${ }^{2}$ Yale Cardiovascular Research Center and Section of Cardiovascular Medicine, Dept. of Internal \\ Medicine, Yale University School of Medicine, New Haven, CT, 06511, USA
}

\section{Abstract}

Segregation of two axial vessels, the dorsal aorta and caudal vein, is one of the earliest patterning events occur during development of vasculature. Despite the importance of this process and recent advances in our understanding on vascular patterning during development, molecular mechanisms that coordinate the segregation of axial vessels remain largely elusive. In this report, we find that Vascular Endothelial Growth Factor-A (Vegf-A) signaling regulates the segregation of dorsal aorta and axial vein during development. Inhibition of Vegf-A pathway components including ligand Vegf-A and its cognate receptor Kdrl, caused failure in segregation of axial vessels in zebrafish embryos. Similarly, chemical inhibition of Mitogen-activated protein kinase kinase (Map2k1)/Extracellular-signal-regulated kinases (Erk) and Phosphatidylinositol 3-kinases (PI3K), which are downstream effectors of Vegf-A signaling pathway, led to the fusion of two axial vessels. Moreover, we find that restoring Erk activity by over-expression of constitutively active MEK in embryos with a reduced level of Vegf-A signaling can rescue the defects in axial vessel segregation. Taken together, our data show that segregation of axial vessels requires the function of Vegf-A signaling, and Erk may function as the major downstream effector in this process.

\section{Keywords}

Vascular Development; zebrafish; Vegf-A; Erk; axial vessel

\section{INTRODUCTION}

The vascular network, which provides the essential functions of delivering oxygen and removing metabolic waste products, is one of the first organs to emerge in embryos. In vertebrates, the vascular system is formed by two distinct processes, vasculogenesis, de novo formation of blood vessels, and angiogenesis, the formation of new blood vessels from preexisting vessels. During development, endothelial cells, the innermost lining of the

\footnotetext{
(C) 2012 Elsevier Inc. All rights reserved.

${ }^{3}$ To whom correspondence should be addressed. suk-won.jin@yale.edu.

*these authors contributed equally to this work
}

Publisher's Disclaimer: This is a PDF file of an unedited manuscript that has been accepted for publication. As a service to our customers we are providing this early version of the manuscript. The manuscript will undergo copyediting, typesetting, and review of the resulting proof before it is published in its final citable form. Please note that during the production process errors may be discovered which could affect the content, and all legal disclaimers that apply to the journal pertain. 
vascular network, form transient aggregates known as the vascular cord in the midline [1,2]. Endothelial cells within the vascular cord start to express arterial and venous specific markers as early as E11.5 in mouse [3], and as early as 18 hours post-fertilization (hpf) in zebrafish [1,4], preceding the emergence of morphologically distinct arteries and veins [5].

Subsequently, developing vasculature acquires the stereotypic hierarchy; arteries and veins are physically separated and only connected by the capillaries. Failure to segregate arteries and veins results in ectopic shunts connecting these two types of vessels, a pathological condition known as arteriovenous malformation (AVM) [6]. While the etiology of AVM remains largely unknown, recent advances start to unravel the molecular and cellular mechanisms that underlie the sorting of arterial and venous endothelial cells. Attenuation of several genes has shown to cause failure in segregation of axial vessels in vertebrates. For instance, mutation in DII4 in mice causes improper segregation of the dorsal aorta and the cardinal vein, creating arteriovenous shunts [7,8]. Similarly, simultaneous reduction of sox 7 and sox 18 genes resulted in improper segregation of the dorsal aorta and the cardinal vein $[9,10,11]$. In all cases, both differentiation of arterial/venous endothelial cells and the segregation of the axial vessels were affected, suggesting that these two events are tightly linked developmental processes.

Recent studies have shown that arterial and venous endothelial cells respond differently to various signaling molecules, which might be the main driving force for the segregation of arteries and veins during development. For instance, Vascular Endothelial Growth Factor-A (Vegf-A) and Vascular Endothelial Growth Factor-C (Vegf-C) signaling preferentially activates dorsal migration of arterial endothelial cells and ventral migration of venous endothelial cells respectively, within the vascular cord, therefore, synergistically promoting the segregation of axial vessels in zebrafish embryos [2]. In addition, the repulsive interaction between EphrinB2 and EphB4 has been shown to be critical in regulating the segregation of arterial endothelial cells from the venous endothelial cells in developing mouse embryo. The dorsal aorta in mice lacking EphrinB2 or EphB4 contained venous endothelial cells, leading to the enlarged dorsal aorta at the expense of the cardinal vein [12]. Later in development, we have recently demonstrated that arterial and venous endothelial cells exhibit different responses to Vegf-A and Bone Morphogenetic Protein (Bmp) signaling [13]. While Bmp signaling induces angiogenesis within venous endothelial cells, arterial endothelial cells remain unaffected. Conversely, Vegf-A signaling preferentially facilitates angiogenesis within arterial endothelial cells without activating venous endothelial cells. Collectively, these reports indicate distinct molecular nature of arterial and venous endothelial cells. However, it is unclear how differences in molecular characteristics of arterial and venous endothelial cells contribute to the segregation of axial vessels. Moreover, it is unknown which signaling pathways regulate the segregation of axial vessels during development. Considering its importance during vascular development, it is likely that VEGF-A signaling may provide a pivotal function in this process.

In this study, we used zebrafish as a model system to investigate the role of Vegf-A signaling during the segregation of axial vessels. We find that reduction in Vegf-A signaling activity led to a failure in the segregation of arteries and veins. Moreover, we find that inhibition of Erk, which are known to function downstream of Vegf-A signaling pathway [4] yield similar defects in developing zebrafish, while activation of Erk in embryos with a reduced level of Vegf-A activity can restore the segregation defects, indicating that activation of Erk by Vegf-A signaling is essential for the proper segregation of axial vessels. Taken together, our data demonstrate the critical role of Vegf-A signaling during the segregation of axial vessels. 


\section{METHODS \& MATERIALS}

\subsection{Zebrafish husbandry and positional cloning}

Zebrafish (Danio rerio) embryos and adults were raised and maintained as previously described under IACUC guidelines [14].

\subsection{Microinjection and chemical treatment}

Morpholinos (MO) (Gene Tools, LLC) were injected into 1-cell-stage $T g(k d r l: E G F P)$ embryos as previously described [1]. The sequences of MOs used in this study are; $k d r l: 5^{\prime}$ CACAAAAAGCGCACACTTACCATGT-3', vegf-aa: $5^{\prime}$ CTCGTCTTATTTCCGTGACTGTTTT- $3^{\prime}$ [15], $k d r: 5^{\prime}-$ GTTTTCTTGATCTCACCTGAACCCT-3', ephrinb2a: 5'CGGTCAAATTCCGTTTCGCGGGA-3'[16], plcy: 5' -

ATTAGCATAGGGAACTTACTTTCG-3' [17]. For chemical treatment, embryos were treated from the 10 somite stage to 30 hours post fertilization (hpf) unless noted otherwise, and fixed overnight in $4 \%$ paraformaldehyde (PFA). To transiently express constitutively active MEK (CA-MEK) [18] in endothelial cells, CA-MEK was subcloned into the Gateway middle entry vector and recombined into $k d r l$ promoter containing vector. The resulting construct allows $k d r l$ promoter regulated, transient expression of CA-MEK in a subset of endothelial cells in injected embryos.

\subsection{Immunohistochemistry, western blot, and in situ hybridization}

Immunohistochemistry and in situ hybridization were performed as previously described [1]. Anti- $\beta$-catenin antibody (1:150, BD Biosciences) was used to show cell boundaries.

\subsection{Phenotypic analyses}

30hpf zebrafish embryos were analyzed by fluorescent stereomicroscope to examine the vascular morphology. For those appear to contain a single axial vessels, transverse sections from $10^{\text {th }}$ somite to $18^{\text {th }}$ somite of $30 \mathrm{hpf}$ zebrafish embryos were analyzed by confocal microscopy. Minimum of ten sections per embryos were analyzed. If the embryo had more than seven sections with single axial vessels, it was counted as 'embryos with a single axial vessel'.

\subsection{Quantitative RT-PCR}

Quantitative RT-PCR was performed as previously reported [19]. The trunk region (above the yolk extension) of the embryos was dissected and RNA was extracted, and pre-tested qRT-PCR primers (Applied biosystems) were used to detect the gene expression.

\section{RESULTS \& DISCUSSION}

To examine the function of Vegf-A signaling during segregation of axial vessels, we attenuated the function of well characterized components of Vegf-A signaling in $T g(k d r l: E G F P)^{s 843}$ embryos [1]. Blocking Vegf-A signaling by injecting morpholino (MO) against vegf-aa or plcy caused failure in segregation of axial vessels (Figure 1A to C), indicating that Vegf-A signaling plays an important role in the segregation of axial vessels.

In zebrafish genome, two functional orthologs of Vascular Endothelial Growth Factor Receptor 2 (VEGFR2/KDR), vegfr2/ $k d r$ and vegfr $4 / k d r l$ are present [20]. Previously, it has been shown that the function of Kdr appears to be largely dispensable for the specification of arterial endothelial cells, although $\mathrm{Kdrl}$ and $\mathrm{Kdr}$ might function redundantly to promote segmental artery formation [21]. Therefore, it is plausible that Kdrl and Kdr may have a distinct role in transducing the activity of Vegf-A signaling during axial vessel segregation 
in zebrafish. To examine this possibility, we selectively inhibited Kdrl or Kdr during development, and analyze the resulting vascular phenotype (Figure 2).

While embryos injected with $k d r l \mathrm{MO}$ contained only a single axial vessel, those injected with $k d r$ MO injected embryos had a distinct dorsal aorta and axial vein, suggesting that the function of Kdr may be dispensable for the segregation of axial vessels (Figure 2B and C). Furthermore, the severity and penetrance of defects on axial vessel segregation in embryos co-injected with $k d r l$ and $k d r \mathrm{MO}$ were comparable to those of embryos injected with $k d r l$ $\mathrm{MO}$ alone, suggesting that the contribution of Kdr to the segregation of axial vessels is likely to be minor.

Interestingly, blocking either Kdrl or Kdr function led a significant reduction in the expression level of arterial and venous specific genes, indicating that both Vegfr2/KDR orthologs are required to promote or maintain the expression of differentiated endothelial cell markers (Figure 2D). Taken together, it seems that both Kdr and Kdrl are required to maintain and/or initiate differentiation of endothelial cells, while Kdrl appears to be the main Vegf-A receptor during segregation of axial vessels.

One of the main downstream effector of Vegf-A signaling is Protein Kinase C (PKC), which is activated by diacylglycerol (DAG) [22]. It has been shown that inhibition of PKC by chemical antagonists blocks the pro-angiogenic effects of Vegf-A signaling. To test whether $\mathrm{PKC}$ is also involved in transducing Vegf-A signaling during segregation of axial vessels, we attenuated the level of PKC activity. 14hpf wild-type zebrafish embryos were treated with $60 \mu \mathrm{M}$ Bisindolylmaleimide, a known antagonist of PKC, for 16 hours [23]. As a positive control, SU5416, a well characterized chemical antagonist of VEGFR2, was used. In embryos treated with SU5416, the axial vessels failed to segregate as expected $(n=20)$ (Figure 3A and E). Similarly, we find that failure in segregation of axial vessels failed in embryos with reduced PKC activity ( $\mathrm{n}=75$ ) (Figure $3 \mathrm{~B}$ and $\mathrm{E}$ ), indicating that $\mathrm{PKC}$ is an essential mediator for Vegf-A signaling during segregation of axial vessels.

Two prominent downstream effectors of PKC activity in the Vegf-A signaling cascade is Map2k1/Erk and Akt/PI3K, which elicit a variety of cellular responses including increased vascular permeability, elevated eNOS production, and initiation of Vegf-A target genes [24]. To determine the function of these two signaling pathways in transducing Vegf-A signaling during the segregation of axial vessels, we also attenuated the activity of Map2k1/Erk or Akt/PI3K, and examined the resulting vascular phenotype.

When embryos were treated with $25 \mu \mathrm{M}$ U0126, a chemical antagonist of Map2k1/Erk [25], from 14 to $30 \mathrm{hpf}$, a significant portion of the embryos had a single axial vessel with impaired circulation, which was reminiscent of the phenotype of embryos with compromised Vegf-A signaling ( $\mathrm{n}=105)$, suggesting that the Map2k1/Erk activity is required to mediate Vegf-A signaling during the segregation of axial vessels (Figure $3 \mathrm{C}$ and $\mathrm{E}$ ).

Similarly, to investigate the contribution of Akt/PI3K in antagonizes Map2k1/Erk in a similar manner to modulate Vegf-A signaling during the segregation of axial vessels, we

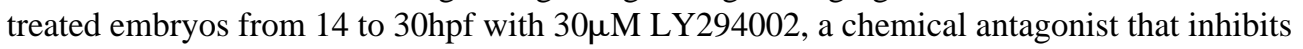
phosphorylation of Akt therefore attenuating PI3K activity ( $n=98)$ [26]. Inhibition of PI3K activity at this stage caused vascular defects comparable to Map2k1/Erk inhibition (Figure 3D and E). Approximately 70 percent of the embryos treated with LY294002 displayed a single axial vessel (Figure 3E). Taken together, our data indicate that effects of Vegf-A signaling during the segregation of the axial vessels require the activity of PKC, Erk, and PI3K. 
To examine whether activation of these downstream effectors is sufficient to initiate segregation of axial vessels in embryos with a reduced level of Vegf-A signaling, we utilized chemical genetics approach. To test whether the activation of PKC is sufficient to bypass the requirement of Vegf-A signaling, $k d r l \mathrm{MO}$ injected embryos were incubated with $16 \mu \mathrm{M}$ 12-O-tetradecanoylphorbol-13-acetate (TPA) [27], a chemical agonist of PKC from 14 to $30 \mathrm{hpf}$. As a positive control, we used GS4012, a chemical agonist of Vegf-A signaling. While $k d r l$ MO injected embryos incubated with DMSO (empty vehicle) contained a single axial vessel ( $\mathrm{n}=40$, Figure 4A and B), 77 percent of $k d r l \mathrm{MO}$ injected embryos treated with GS4012, and 86 percent of $k d r l$ MO injected embryos incubated with TPA had a clearly separate dorsal aorta and axial vein ( $n=55$ for GS4012, and $n=42$ for TPA, Figure 4A and B). Therefore, activation of PKC is sufficient to alleviate defects on the segregation of axial vessels caused by reduced Vegf-A signaling.

To further examine the function of Map2k1/Erk in the segregation of axial vessels, constitutively active MEK (CA-MEK) was selectively expressed in endothelial cells in $k d r l$ MO injected embryos [18] (Figure 4C and D). Since MEK induces activation of Map2k1/ Erk, ectopic expression of CA-MEK in endothelial cells should alleviate the phenotype of $k d r l \mathrm{MO}$ injected embryos. While less than two percent of embryos co-injected with a vector encoding Blue Fluorescent Protein (BFP) and $k d r l \mathrm{MO}$ contained two axial vessels at 28hpf (Figure 4E), a significant portion (38\%) of embryos co-injected with the CA-MEK construct and $k d r l \mathrm{MO}$ had clearly distinguishable axial vessels (Figure 4E), suggesting that Map2k1/ Erk activity is critical for the segregation of the axial vessels, but dispensable for the maintenance of the arterial or venous specific gene expression downstream of Vegfr signaling.

\section{CONCLUSION}

In this report, we investigated the function of Vegf-A signaling in the segregation of axial vessels during vertebrate development. We find that Vegf-A signaling is indispensable for the proper segregation of axial vessels. Among two zebrafish orthologs of VEGFR3/KDR, we find that the function of Kdr appears to be dispensable for the segregation of axial vessels. Despite drastically reduced expression of arterial or venous markers, a distinct dorsal aorta and axial vein were formed in $k d r$ MO injected embryos. Therefore, it appears that $\mathrm{Kdrl}$ is the main receptor to mediate Vegf-A signaling during the segregation of axial vessels. During the segregation of axial vessels, Vegf-A signaling appears to promote activity of Erk and PI3K, since inhibition of either Erk or PI3K activity caused similar vascular defects as in embryos with compromised Vegf-A signaling. Moreover, activation of Erk signaling in Vegf-A signaling deficient embryos restored the defects in axial vessel segregation, indicating that Erk signaling may play a key role in mediating Vegf-A signaling activity in this process.

\section{Acknowledgments}

The authors would like to thank members of Jin lab for helpful discussion. This work has been supported by Developmental Biology Training Grant to C.E.S. and American Heart Association Pre-doctoral Fellowship to M.J.W., American Heart Association Pre-doctoral Fellowship to J.D.K., and grants from the NIH (HL090960) and the American Heart Association Scientist Development Award to S.-W.J.

\section{References}

1. Jin SW, Beis D, Mitchell T, Chen JN, Stainier DY. Cellular and molecular analyses of vascular tube and lumen formation in zebrafish. Development. 2005; 132:5199-5209. [PubMed: 16251212] 
2. Herbert SP, Huisken J, Kim TN, Feldman ME, Houseman BT, Wang RA, Shokat KM, Stainier DY. Arterial-venous segregation by selective cell sprouting: an alternative mode of blood vessel formation. Science. 2009; 326:294-298. [PubMed: 19815777]

3. Adams RH, Wilkinson GA, Weiss C, Diella F, Gale NW, Deutsch U, Risau W, Klein R. Roles of ephrinB ligands and EphB receptors in cardiovascular development: demarcation of arterial/venous domains, vascular morphogenesis, and sprouting angiogenesis. Genes Dev. 1999; 13:295-306. [PubMed: 9990854]

4. Hong CC, Peterson QP, Hong JY, Peterson RT. Artery/vein specification is governed by opposing phosphatidylinositol-3 kinase and MAP kinase/ERK signaling. Curr Biol. 2006; 16:1366-1372. [PubMed: 16824925]

5. Jin SW, Patterson C. The opening act: vasculogenesis and the origins of circulation. Arterioscler Thromb Vasc Biol. 2009; 29:623-629. [PubMed: 19008532]

6. Leblanc GG, Golanov E, Awad IA, Young WL. Biology of vascular malformations of the brain. Stroke. 2009; 40:e694-702. [PubMed: 19834013]

7. Duarte A, Hirashima M, Benedito R, Trindade A, Diniz P, Bekman E, Costa L, Henrique D, Rossant J. Dosage-sensitive requirement for mouse Dll4 in artery development. Genes Dev. 2004; 18:24742478. [PubMed: 15466159]

8. Gale NW, Dominguez MG, Noguera I, Pan L, Hughes V, Valenzuela DM, Murphy AJ, Adams NC, Lin HC, Holash J, Thurston G, Yancopoulos GD. Haploinsufficiency of delta-like 4 ligand results in embryonic lethality due to major defects in arterial and vascular development. Proc Natl Acad Sci U S A. 2004; 101:15949-15954. [PubMed: 15520367]

9. Pendeville H, Winandy M, Manfroid I, Nivelles O, Motte P, Pasque V, Peers B, Struman I, Martial JA, Voz ML. Zebrafish Sox7 and Sox18 function together to control arterial-venous identity. Dev Biol. 2008; 317:405-416. [PubMed: 18377889]

10. Herpers R, van de Kamp E, Duckers HJ, Schulte-Merker S. Redundant roles for sox7 and sox 18 in arteriovenous specification in zebrafish. Circ Res. 2008; 102:12-15. [PubMed: 18032732]

11. Cermenati S, Moleri S, Cimbro S, Corti P, Del Giacco L, Amodeo R, Dejana E, Koopman P, Cotelli F, Beltrame M. Sox18 and Sox7 play redundant roles in vascular development. Blood. 2008; 111:2657-2666. [PubMed: 18094332]

12. Kim YH, Hu H, Guevara-Gallardo S, Lam MT, Fong SY, Wang RA. Artery and vein size is balanced by Notch and ephrin B2/EphB4 during angiogenesis. Development. 2008; 135:37553764. [PubMed: 18952909]

13. Wiley DM, Kim JD, HJ, Hong CC, Bautch VL, Jin SW. Distinct Signaling Pathways Regulate Sprouting Angiogenesis from the Dorsal Aorta and Axial Vein. Nat Cell Biol. 2011

14. Westerfield, M. The zebrafish book, A Guide for the laboratory use of zebrafish (Danio rerio). 4. University of Oregon Press; Eugene: 2000.

15. Nasevicius A, Ekker SC. Effective targeted gene 'knockdown' in zebrafish. Nat Genet. 2000; 26:216-220. [PubMed: 11017081]

16. Cooke JE, Kemp HA, Moens CB. EphA4 is required for cell adhesion and rhombomere-boundary formation in the zebrafish. Curr Biol. 2005; 15:536-542. [PubMed: 15797022]

17. Lawson ND, Mugford JW, Diamond BA, Weinstein BM. phospholipase C gamma-1 is required downstream of vascular endothelial growth factor during arterial development. Genes Dev. 2003; 17:1346-1351. [PubMed: 12782653]

18. Scholl FA, Dumesic PA, Khavari PA. Mek1 alters epidermal growth and differentiation. Cancer Res. 2004; 64:6035-6040. [PubMed: 15342384]

19. Lee CY, Vogeli KM, Kim SH, Chong SW, Jiang YJ, Stainier DY, Jin SW. Notch signaling functions as a cell-fate switch between the endothelial and hematopoietic lineages. Curr Biol. 2009; 19:1616-1622. [PubMed: 19747827]

20. Bussmann J, Lawson N, Zon L, Schulte-Merker S. Zebrafish VEGF receptors: a guideline to nomenclature. PLoS Genet. 2008; 4:e1000064. [PubMed: 18516225]

21. Covassin LD, Villefranc JA, Kacergis MC, Weinstein BM, Lawson ND. Distinct genetic interactions between multiple Vegf receptors are required for development of different blood vessel types in zebrafish. Proc Natl Acad Sci U S A. 2006; 103:6554-6559. [PubMed: 16617120] 
22. Hong CC, Kume T, Peterson RT. Role of crosstalk between phosphatidylinositol 3-kinase and extracellular signal-regulated kinase/mitogen-activated protein kinase pathways in artery-vein specification. Circ Res. 2008; 103:573-579. [PubMed: 18796644]

23. Toullec D, Pianetti P, Coste H, Bellevergue P, Grand-Perret T, Ajakane M, Baudet V, Boissin P, Boursier E, Loriolle F, et al. The bisindolylmaleimide GF 109203X is a potent and selective inhibitor of protein kinase C. J Biol Chem. 1991; 266:15771-15781. [PubMed: 1874734]

24. Zachary I. VEGF signalling: integration and multi-tasking in endothelial cell biology. Biochem Soc Trans. 2003; 31:1171-1177. [PubMed: 14641020]

25. Favata MF, Horiuchi KY, Manos EJ, Daulerio AJ, Stradley DA, Feeser WS, Van Dyk DE, Pitts WJ, Earl RA, Hobbs F, Copeland RA, Magolda RL, Scherle PA, Trzaskos JM. Identification of a novel inhibitor of mitogen-activated protein kinase kinase. J Biol Chem. 1998; 273:18623-18632. [PubMed: 9660836]

26. Vlahos CJ, Matter WF, Hui KY, Brown RF. A specific inhibitor of phosphatidylinositol 3-kinase, 2-(4-morpholinyl)-8-phenyl-4H-1-benzopyran-4-one (LY294002). J Biol Chem. 1994; 269:52415248. [PubMed: 8106507]

27. Bell RM, Hannun YA, Loomis CR. Mechanism of regulation of protein kinase C by lipid second messengers. Symp Fundam Cancer Res. 1986; 39:145-156. [PubMed: 3321305] 


\section{Highlights}

1. VEGF-A signaling regulates the segregation of axial vessels.

2. VEGF-A signaling is mediated by PKC and ERK in this process.

3. Ectopic activation of ERK is sufficient to rescue defects in vessel segregation. 


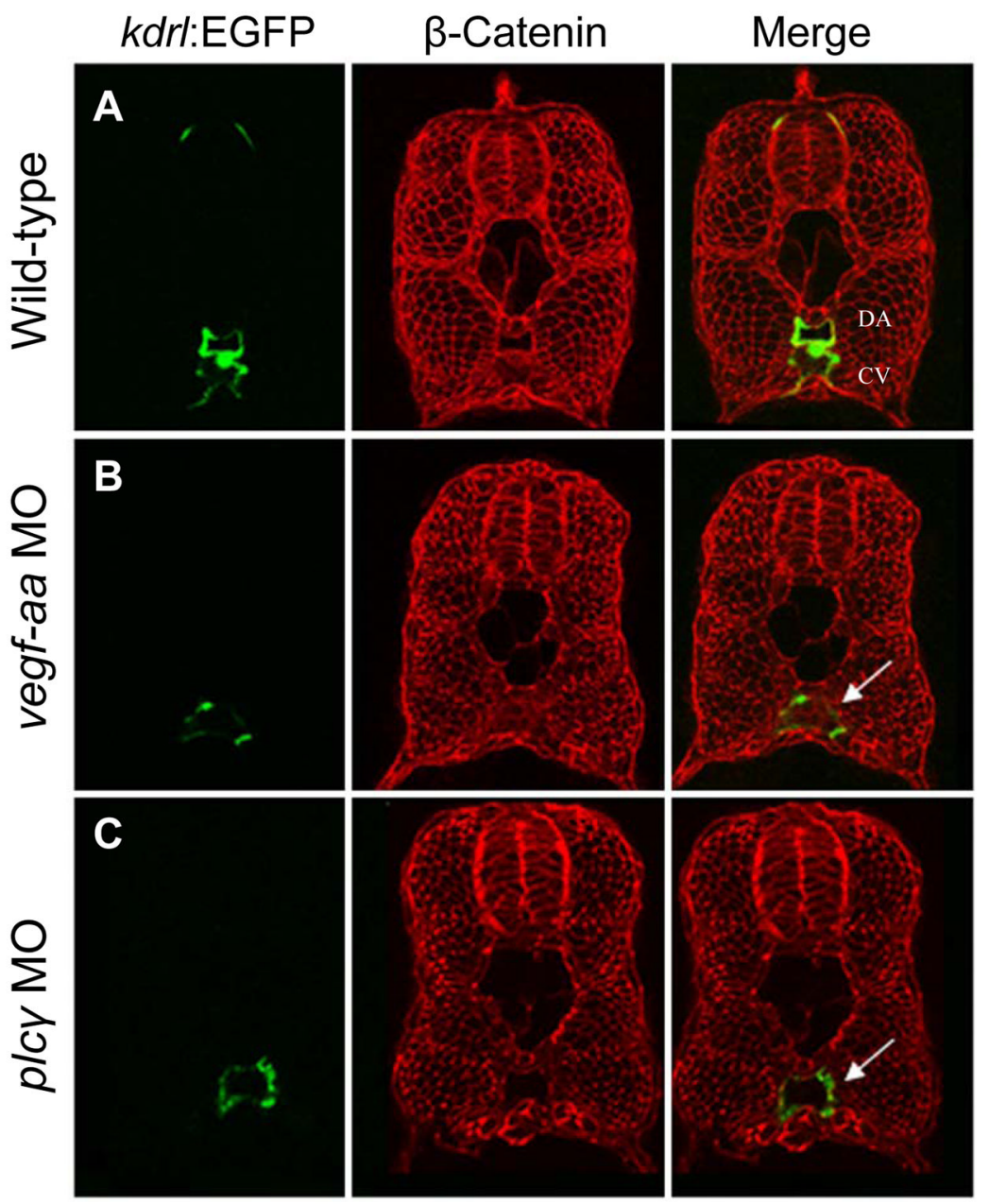

Figure 1. Attenuation of Vegf-A signaling components causes defects in axial vessel segregation as observed in $k d r l^{s 828}$ mutants

Transverse sections taken at the level of the $14^{\text {th }}$ somite were analyzed by confocal microscopy. 36hpf wild-type (A), vegf-aa MO injected (B), and plcy MO injected (C) embryos in $T g(k d r l: E G F P)^{s 843}$ background. Endothelial cells are visualized by the expression of EGFP transgene (green), cell boundaries are shown by Anti- $\beta$-catenin staining (red). White arrows in panels $\mathbf{B}$ and $\mathbf{C}$ point to the single axial vessel. Abbreviations: DA, dorsal aorta, $\mathrm{CV}$, caudal vein. 

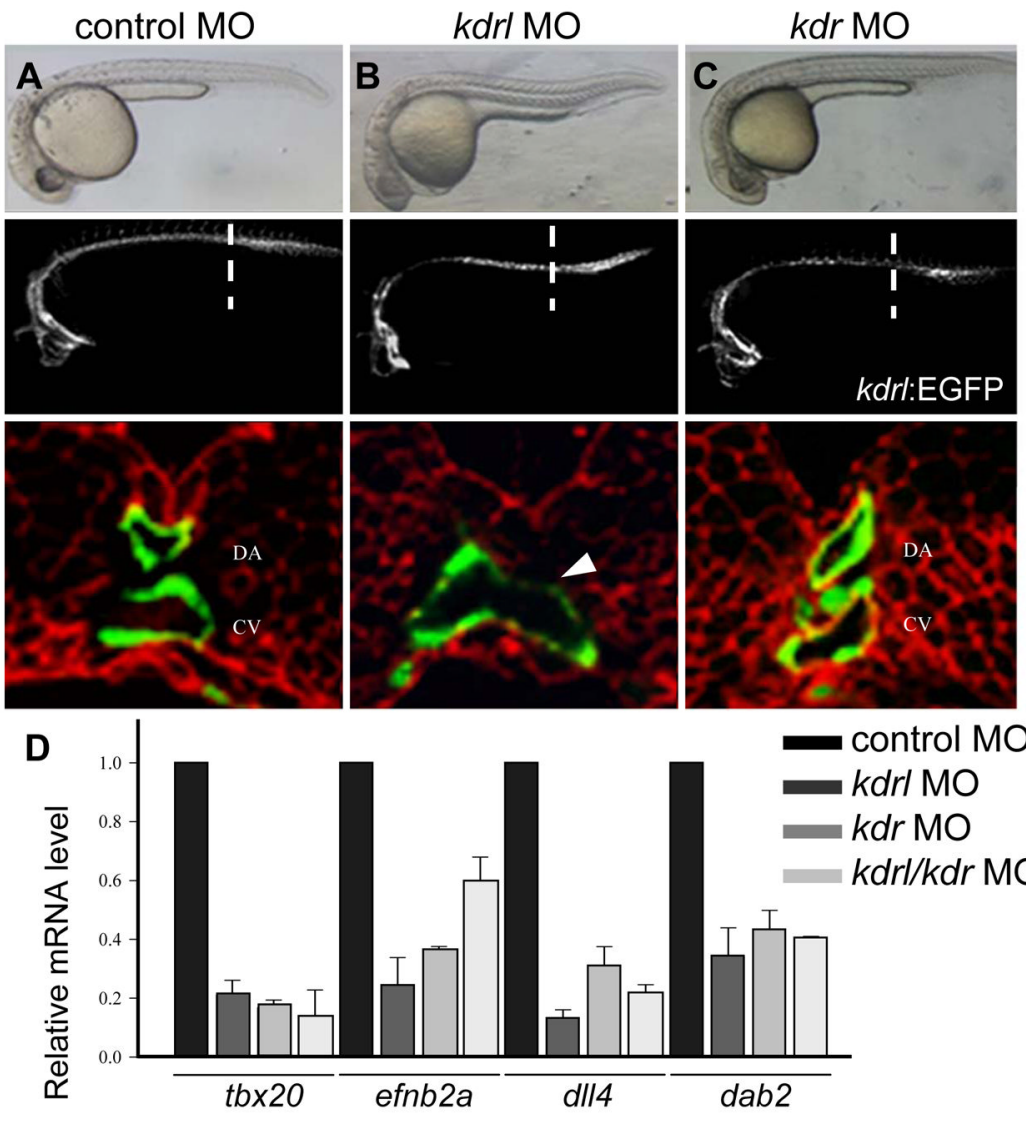

Figure 2. Kdrl functions as the main receptor for Vegf-A signaling during the segregation of axial vessels

Bright field micrographs (top row), epifluorescence micrographs (middle row), and confocal images of a transverse section at the $14^{\text {th }}$ somite level (bottom row) of 36hpf embryos injected with control MO (A), $k d r l \mathrm{MO}(\mathbf{B})$, or $k d r \mathrm{MO}(\mathbf{C})$ in $T g(k d r l \text { :EGFP })^{843}$ background. Endothelial cells are visualized by the expression of EGFP transgene (green), cell boundaries are shown by Anti- $\beta$-catenin staining (red). White arrowhead in panel B points to the single axial vessel. (D) Quantitative RT-PCR of endothelial specific genes in 36hpf MO injected embryos. Attenuation of either $k d r l$ or $k d r$ significantly reduced the expression of arterial and venous marker expression. Abbreviations: DA, dorsal aorta, CV, caudal vein. 

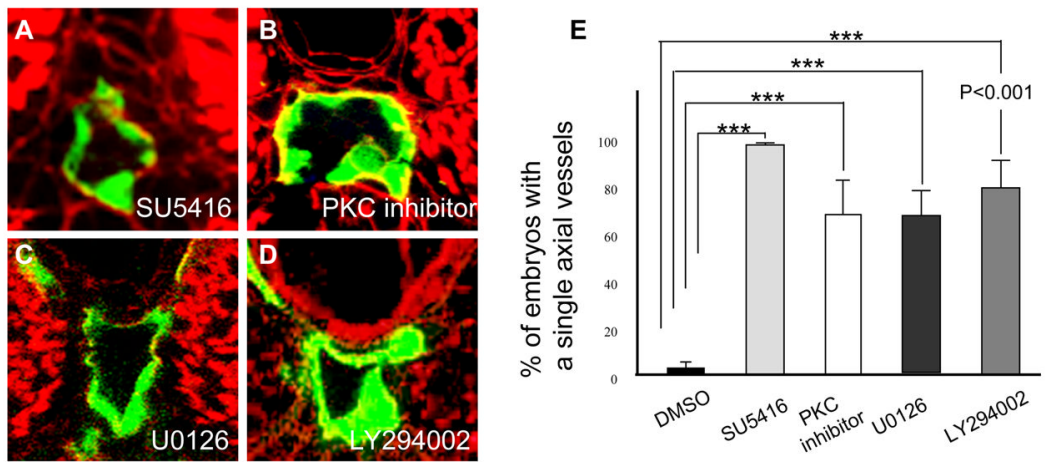

Figure 3. Attenuation of Vegf-A downstream effectors caused defects in segregation of axial vessels

Transverse sections taken at the level of the $14^{\text {th }}$ somite were analyzed by confocal microscopy. 36hpf $T g(k d r l \text { :EGFP })^{843}$ embryos treated with $1 \mu \mathrm{M}$ SU5416 (A), $60 \mu \mathrm{M}$ Bisindolylmaleimide, a chemical antagonist of PKC (B), 25 $\mu \mathrm{M} \mathrm{U0126}(\mathbf{C})$, or 30 $\mu \mathrm{M}$ LY294002 (D). (E) Quantification of chemical treatments. Compared to DMSO treated embryos, the percentage of embryos with a single axial vessel was significantly increased in embryos treated with SU5416, Bisindolylmaleimide, U0126, or LY294002 ( P<0.001). 


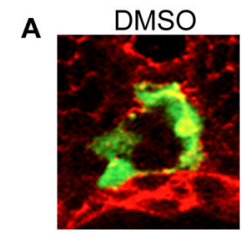

B

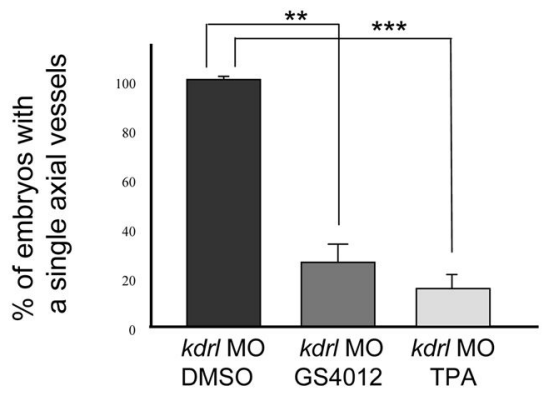

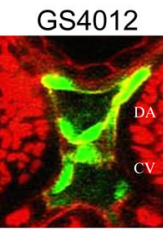

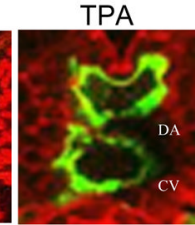

DMSO GS4012 TPA

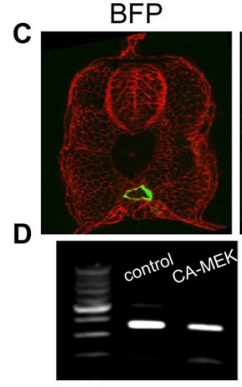

$k d r l \mathrm{MO}$

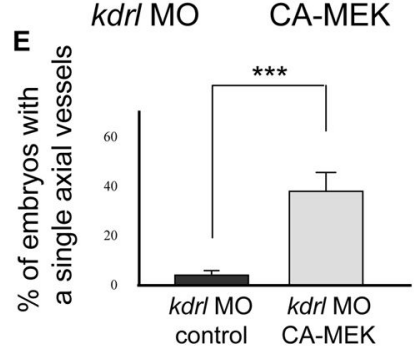

Figure 4. Activation of PKC or Erk activity can rescue the defects in segregation of axial vessels in embryos with compromised Vegf-A signaling

Transverse sections taken at the level of the $14^{\text {th }}$ somite were analyzed by confocal microscopy. 36hpf $T g\left(k d r l\right.$ :EGFP) ${ }^{s 43}$ embryos treated with DMSO (left), $7.5 \mu \mathrm{g} / \mathrm{ml}$ GS4012 (middle), and 16 $\mathrm{M}$ 12-O-tetradecanoylphorbol-13-acetate (right). (B) Treatment with $16 \mu \mathrm{M} 12-\mathrm{O}$-tetradecanoylphorbol-13-acetate restored the defects in segregation of axial vessels better than GS4012, a positive control. (C) Transverse sections taken at the level of the $14^{\text {th }}$ somite from $k d r l$ MO injected embryos, co-injected with either vector containing Blue Fluorescent Protein (BFP) or constitutively active MEK (CA-MEK). (D) PCR confirmation showing the validity of $k d r l \mathrm{MO}$ (left) and expression of CA-MEK construct (right). (E) The defect in axial vessel segregation in $k d r l \mathrm{MO}$ injected embryos was substantially rescued by ectopic activation of MEK, which can increase the level of Erk activity. Abbreviations: DA, dorsal aorta, CV, caudal vein. 